\title{
General Schlesinger Systems and Their Symmetry from the View Point of Twistor theory
}

Hironobu Kimura, Damiran Tseveenamijil

To cite this article: Hironobu Kimura, Damiran Tseveenamijil (2013) General Schlesinger Systems and Their Symmetry from the View Point of Twistor theory, Journal of Nonlinear Mathematical Physics 20: Supplement 1, 130-152, DOI:

https://doi.org/10.1080/14029251.2013.862441

To link to this article: https://doi.org/10.1080/14029251.2013.862441

Published online: 04 January 2021 


\title{
General Schlesinger Systems and Their Symmetry from the View Point of Twistor theory
}

\author{
Hironobu Kimura \\ Department of Mathematics, Kumamoto University, Kurokami 2-39-1 \\ Kumamoto 8555, Japan \\ hiro@sci.kumamoto-u.ac.jp \\ Damiran Tseveenamijil \\ School of Economics and Business, Mongolian State University of Agriculture \\ Zaisan-17024, Ulanbaataar, Mongolia \\ tsezulaa@yahoo.com \\ Received 3 september 2012 \\ Accepted 28 May 2013
}

\begin{abstract}
Isomonodromic deformation of linear differential equations on $\mathbb{P}^{1}$ with regular and irregular singular points is considered from the view point of twistor theory. We give explicit form of isomonodromic deformation using the maximal abelian subgroup $H$ of $G=\mathrm{GL}_{N+1}(\mathbb{C})$ which appeared in the theory of general hypergeometric functions on a Grassmannian manifold. This formulation enables us to obtain a group of symmetry for the nonlinear system which is an Weyl group analogue $N_{G}(H) / H$.
\end{abstract}

Keywords: Isomonodromic deformation; Twistor theory; Schlesinger system.

2000 Mathematics Subject Classification: 34M55, 34M56, 32L25

\section{Introduction}

The Schlesinger system is a completely integrable system

$$
\frac{\partial A_{j}}{\partial t_{i}}=\frac{\left[A_{i}, A_{j}\right]}{t_{i}-t_{j}} \quad(i \neq j), \quad \frac{\partial A_{i}}{\partial t_{i}}=-\sum_{j \neq i} \frac{\left[A_{i}, A_{j}\right]}{t_{i}-t_{j}}
$$

for the $r \times r$ matrix unknowns $A_{0}, A_{1}, \ldots, A_{N}$ satisfying

$$
A_{0}+A_{1}+\cdots+A_{N}=0
$$

where $\left[A_{i}, A_{j}\right]=A_{i} A_{j}-A_{j} A_{i}$. This system was obtained by L. Schlesinger [12] as the system describing the isomonodromic deformation of a Fuchsian system

$$
\frac{d y}{d \zeta}=\left(\sum_{j=0}^{N} \frac{A_{j}(t)}{\zeta-t_{j}}\right) y .
$$

In the case $N=3, r=2$ and $t_{0}=\infty, t_{1}=0, t_{2}=1, t_{3}=t$, the Schlesinger system is written as

$$
\frac{d A_{1}}{d t}=\frac{\left[A_{3}, A_{1}\right]}{t}, \quad \frac{d A_{2}}{d t}=\frac{\left[A_{3}, A_{2}\right]}{t-1}, \quad \frac{d A_{3}}{d t}=-\frac{\left[A_{3}, A_{1}\right]}{t}-\frac{\left[A_{3}, A_{2}\right]}{t-1},
$$


and is known to be intimately related with the second order differential equation called the sixth Painlevé equation $P_{V I}$. In fact, it is explained in [3] that if we define $q$ from a solution $\left(A_{1}(t), A_{2}(t), A_{3}(t)\right)$ of (1.3) by

$$
q=\frac{t\left(A_{1}\right)_{12}}{(t+1)\left(A_{1}\right)_{12}+t\left(A_{2}\right)_{12}+\left(A_{3}\right)_{12}}
$$

where $\left(A_{j}\right)_{12}$ is the $(1,2)$-entry of $A_{j}$, then $q$ satisfies $P_{V I}$. Recall that the Painlevé equations $P_{J}(J=$ $I, \ldots, V I)$ are the second order nonlinear differential equations of the form

$$
q^{\prime \prime}=R\left(t, q, q^{\prime}\right), \quad R \in \mathbb{C}\left(t, q, q^{\prime}\right), \quad '=\frac{d}{d t},
$$

which form representatives of classification, by birational transformations, of Eq. (1.4) enjoying the Painlevé property, namely, any solution, considered as a function of $t$ and initial conditions, has no branch point which changes its position according as the variation of initial conditions [1, 11]. The Painlevé equations appear and play important roles in many fields of mathematics and mathematical physics, and now recognized as nonlinear differential equations defining new special functions [2,9,13]. Among the Painlevé equations, the master one is $P_{V I}$ and the others are derived by "degeneration" according to the diagram:

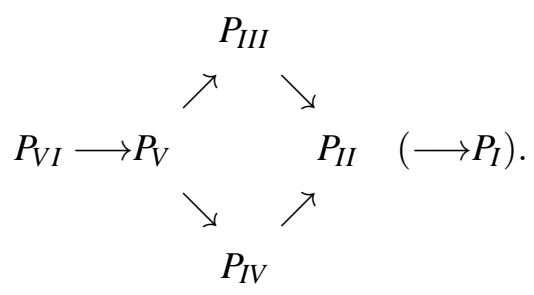

Here the arrow $P_{V I} \rightarrow P_{V}$ means, for example, that we can get $P_{V}$ from $P_{V I}$ by the change $(t, q) \mapsto(\tilde{t}, \tilde{q}): t=1+\varepsilon \tilde{t}, q=\tilde{q}$, a suitable change of parameters in $P_{V I}$ and by taking a limit $\varepsilon \rightarrow 0$, see [3], p143. As $P_{V I}$ is related with the Schlesinger system (1.3), we know that the other Painlevé equations are also related with the systems analogous to (1.3), which are obtained by isomonodromic deformation of non Fuchsian systems on $\mathbb{P}^{1}$.

The aim of this paper is to describe nonlinear systems obtained from the Schlesinger system by degeneration (or the corresponding isomonodromic deformations) in explicit manner, which we call the general Schlesinger systems (Def. 3.1) in this paper. We follow the idea of Mason and Woodhouse [6]. Their idea is to derive the general Schlesinger systems as the systems describing particular solutions of generalized anti-self-dual Yang-Mills equation (GASDYM) on the complex Grassmannian manifold $G_{2, N+1}$ of 2-dimensional subspaces of $\mathbb{C}^{N+1}$.

Let us explain further our motivation and what we want to achieve. Originally, the partitions of 4 are associated with the Painlevé equations (or with the corresponding general Schlesinger systems):

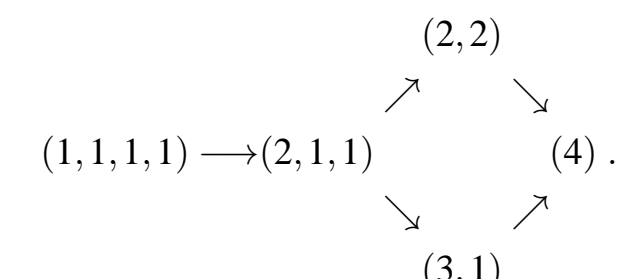


For example, the partition $(1,1,1,1)$ for $P_{V I}$ means that the linear system of differential equations, whose isomonodromic deformation provides $P_{V I}$, has 4 regular singular points $x=0,1, t, \infty$ (see the explanation just above (1.3)). Likewise, the partition $(2,1,1)$ for $P_{V}$ means that this equation is obtained from the isomonodromic deformation of a linear system with one irregular singular point of Poincaré rank 1 and two regular singular points.

It is to be noted that each Painlevé equation has particular solutions expressed in terms of hypergeometric type function ([3]):

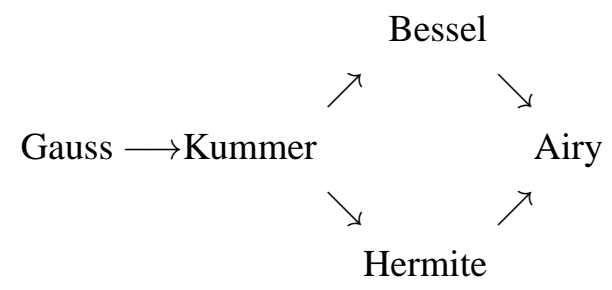

and that the partitions of 4 in (1.6) are already attached to these hypergeometric type functions in the context of general hypergeometric functions (GHF) on the Grassmannian manifold [4]. In this theory, a partition $\lambda$ specifies a type of regular elements of $\mathrm{GL}_{4}(\mathbb{C})$ and the $\mathrm{GHF}$ is constructed using the maximal abelian subgroup $H_{\lambda}$ of $\mathrm{GL}_{4}(\mathbb{C})$ obtained as the centralizer of a regular element of type $\lambda$, see Sec. 2 about $H_{\lambda}$. It was surprising that the same maximal abelian subgroups were used in the work of Mason and Woodhouse [5] to derive the the general Schlesinger systems, corresponding to Painlevé equations, as the subsystems of $s l_{2}(\mathbb{C})$ anti-self-dual Yang-Mills equation on $G_{2,4}$ invariant by the action of the group $H_{\lambda}$. Moreover they also showed in [6] that the Schlesinger system (1.1) can be obtained from GASDYM on the Grassmannian manifold $G_{2, N+1}$ by considering the holomorphic vector bundle on an open subset of the twistor space $\mathbb{P}^{N}$, coming from a solution of GASDYM by Ward correspondence, equivariant under the action of $H_{(1, \ldots, 1)}$. They also mentioned that the degenerated systems can be obtained in a similar way using $H_{\lambda}$. Unfortunately, they did not give an explicit form of isomonodromic deformations and the corresponding general Schlesinger systems.

In this paper we make more explicit the construction of [6] for general Schlesinger systems for an arbitrary partition $\lambda$ of $N+1$ and discuss their group of symmetry as an analogue of Weyl group.

\section{Regular elements and Maximal abelian subgroups}

Let $G=\mathrm{GL}_{N+1}(\mathbb{C})$ be the complex general linear group of matrices of size $N+1$. For $g \in G$, let $\operatorname{Ad}_{g}: G \rightarrow G$ be defined by $a \mapsto \operatorname{Ad}_{g}(a)=g a g^{-1}$, which gives the adjoint action of $G$ on itself. Denote the orbit of $a \in G$ by $O(a)=\left\{\operatorname{Ad}_{g}(a) \mid g \in G\right\}$ and the centralizer of $a \in G$ by $Z_{G}(a)=$ $\left\{g \in G \mid \operatorname{Ad}_{g}(a)=a\right\}$. We know that both $O(a)$ and $Z_{G}(a)$ are complex manifolds and $\operatorname{dim}_{\mathbb{C}} G=$ $\operatorname{dim}_{\mathbb{C}} O(a)+\operatorname{dim}_{\mathbb{C}} Z_{G}(a)$ holds.

Definition 2.1. An element $a \in G$ is said to be a regular element if $\operatorname{dim} O(a)$ is maximum, in other word, $\operatorname{dim} Z_{G}(a)$ is minimum.

It is seen that $\operatorname{dim} Z_{G}(a)=N+1$ if $a$ is a regular element (see below). It is known and is easy to see that $a \in G$ is a regular element iff the Jordan cells of the Jordan normal form of $a$ have distinct 
eigenvalues, i.e., for some partition $\lambda=\left(n_{1}, \ldots, n_{\ell}\right)$ of $N+1, a$ is conjugate to

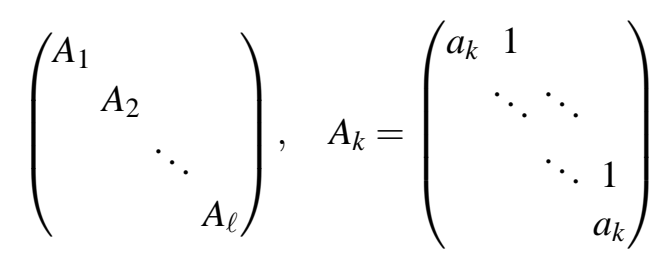

with distinct $a_{1}, \ldots, a_{\ell}$, where $A_{k} \in \mathrm{GL}_{n_{k}}(\mathbb{C})$.

What we concern is the groups obtained as centralizers of regular elements, which are given explicitly as follows. When $a \in G$ itself is the Jordan normal form as in (2.1), then

$$
Z_{G}(a)=\left\{\left(\begin{array}{ccc}
h^{(1)} & & \\
& \ddots & \\
& & h^{(\ell)}
\end{array}\right) \mid h^{(k)} \in J\left(n_{k}\right)\right\}
$$

where $J(n)$ is the abelian subgroup of $\mathrm{GL}_{n}(\mathbb{C})$ of the form

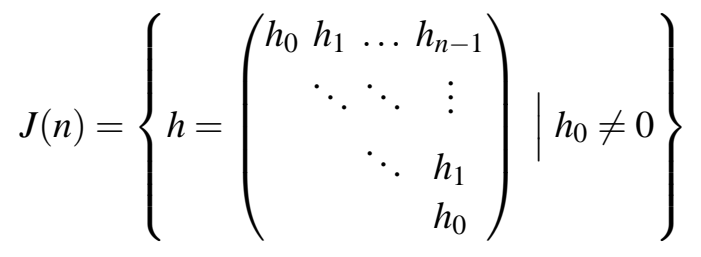

called $n$-dimensional Jordan group. We also write $h$ as

$$
h=h_{0} I+h_{1} \Lambda+\cdots+h_{n-1} \Lambda^{n-1}
$$

using the shift matrix $\Lambda=\left(\delta_{i+1, j}\right)_{0 \leq i, j<n}$ of size $n$. The group $Z_{G}(a)$, which is isomorphic to the product group $J\left(n_{1}\right) \times \cdots \times J\left(n_{\ell}\right)$, and is irrelevant to the eigenvalues, will be denoted as $H_{\lambda}$ so as to emphasize that the group is determined by the partition $\lambda$.

Let $\mathfrak{j}(n)$ and $\mathfrak{h}_{\lambda}$ be the Lie algebras of $J(n)$ and $H_{\lambda}$, respectively:

$$
\mathfrak{j}(n)=\left\{\xi=\xi_{0} I+\xi_{1} \Lambda+\cdots+\xi_{n-1} \Lambda^{n-1} \mid \xi_{i} \in \mathbb{C}\right\} \simeq \mathbb{C}^{n}
$$

and

$$
\mathfrak{h}_{\lambda}=\left\{\left(\begin{array}{ccc}
\xi^{(1)} & & \\
& \ddots & \\
& & \xi^{(\ell)}
\end{array}\right) \mid \xi^{(k)} \in \mathfrak{j}\left(n_{k}\right)\right\} \simeq \mathfrak{j}\left(n_{1}\right) \oplus \cdots \oplus \mathfrak{j}\left(n_{\ell}\right) .
$$

In order to make explicit the relation between $H_{\lambda}$ and its Lie algebra $\mathfrak{h}_{\lambda}$, we introduce the following functions. 
Definition 2.2. Let $T$ be an indeterminate. Define the functions $\theta_{m}(x)$ of $x=\left(x_{0}, x_{1}, \ldots\right)$ by

$$
\log \left(x_{0}+x_{1} T+x_{2} T^{2}+\cdots\right)=\sum_{m=0}^{\infty} \theta_{m}(x) T^{m}
$$

Since

$$
\begin{aligned}
& \log \left(x_{0}+x_{1} T+x_{2} T^{2}+\cdots\right) \\
& =\log x_{0}+\sum_{m=1}^{\infty} \frac{(-1)^{m+1}}{m}\left(\frac{x_{1}}{x_{0}} T+\frac{x_{2}}{x_{0}} T^{2}+\cdots\right)^{m},
\end{aligned}
$$

we see that $\theta_{0}(x)=\log x_{0}$ and, for $m \geq 1$,

$$
\theta_{m}(x)=\sum(-1)^{k_{1}+\cdots+k_{m}-1} \frac{\left(k_{1}+\cdots+k_{m}-1\right) !}{k_{1} ! \cdots k_{m} !}\left(\frac{x_{1}}{x_{0}}\right)^{k_{1}} \cdots\left(\frac{x_{m}}{x_{0}}\right)^{k_{m}},
$$

where the sum is taken over all $\left(k_{1}, \ldots, k_{m}\right) \in \mathbb{Z}_{\geq 0}^{m}$ satisfying $k_{1}+2 k_{2}+\cdots+m k_{m}=m$. For example first few of them are

$$
\begin{aligned}
& \theta_{0}(x)=\log x_{0}, \\
& \theta_{1}(x)=\frac{x_{1}}{x_{0}} \\
& \theta_{2}(x)=\frac{x_{2}}{x_{0}}-\frac{1}{2}\left(\frac{x_{1}}{x_{0}}\right)^{2}, \\
& \theta_{3}(x)=\frac{x_{3}}{x_{0}}-\left(\frac{x_{1}}{x_{0}}\right)\left(\frac{x_{2}}{x_{0}}\right)+\frac{1}{3}\left(\frac{x_{1}}{x_{0}}\right)^{3}, \\
& \theta_{4}(x)=\frac{x_{4}}{x_{0}}-\frac{1}{2}\left\{\left(\frac{x_{2}}{x_{0}}\right)^{2}+2\left(\frac{x_{1}}{x_{0}}\right)\left(\frac{x_{3}}{x_{0}}\right)\right\}+\left(\frac{x_{1}}{x_{0}}\right)^{2}\left(\frac{x_{2}}{x_{0}}\right)-\frac{1}{4}\left(\frac{x_{1}}{x_{0}}\right)^{4} .
\end{aligned}
$$

From these explicit form we see that $\theta_{m}(x), m \geq 1$, has a pole along $x_{0}=0$ of order $m$ and is a weighted homogeneous polynomial of $x_{1} / x_{0}, \ldots, x_{m} / x_{0}$ of weight $m$ when the weight of $x_{i}$ is set to be $i$.

Let $\tilde{J}(n)$ be the universal covering group of $J(n)$. Then we see that $\log : \tilde{J}(n) \rightarrow \mathfrak{j}(n)$ defined by

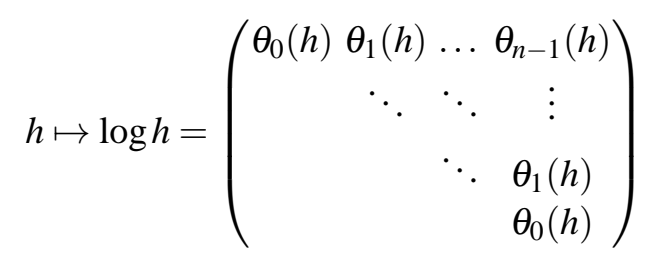

gives a biholomorphic map. To invert the map

$$
x=\left(x_{0}, x_{1}, \ldots\right) \mapsto \xi=\left(\xi_{0}, \xi_{1}, \ldots\right)=\left(\theta_{0}(x), \theta_{1}(x), \ldots\right),
$$

we take the exponential of (2.4) and define the polynomials $p_{m}(\xi)$ by

$$
\exp \left(\xi_{0}+\xi_{1} T+\cdots\right)=e^{\xi_{0}} \sum_{m=0}^{\infty} p_{m}(\xi) T^{m}
$$


where $p_{m}(\xi)$ is the $m$-th Schur polynomial of $\left(\xi_{1}, \xi_{2}, \ldots\right)$ corresponding to the partition $(m)$ :

$$
p_{m}(\xi)=\sum_{k_{1}+2 k_{2}+\cdots+m k_{m}=m} \frac{\xi_{1}^{k_{1}} \cdots \xi_{m}^{k_{m}}}{k_{1} ! \cdots k_{m} !} .
$$

The map

$$
\mathfrak{j}(n) \ni \xi \mapsto \exp \xi=e^{\xi_{0}} \sum_{m=0}^{n-1} p_{m}(\xi) \Lambda^{m}
$$

defines exp $: \mathfrak{j}(n) \rightarrow J(n)$.

\section{Main Results}

We state the main results of this paper, which give the explicit expression for the results in $[5,6]$ concerning the isomonodromic deformation of linear ordinary differential equations on $\mathbb{P}^{1}$ treated from the twistor theoretic point of view. The basic geometric picture is the double fibration

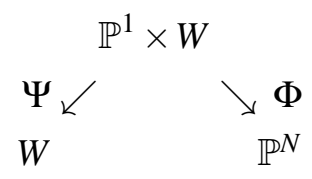

where $W$ is a certain open subset of Grassmannian manifold $\mathrm{Gr}_{2, N+1}$, which is represented as the quotient space $\mathrm{GL}_{2}(\mathbb{C}) \backslash Z$ of the set $Z$ of $2 \times(N+1)$ complex matrices of rank 2 by the right action of $\mathrm{GL}_{2}(\mathbb{C})$. Here the map $\Psi$ is the projection to the second factor and $\Phi$ is defined by $([t],[z]) \mapsto$ $[t z],[t] \in \mathbb{P}^{1}$ being a point with its homogeneous coordinates $t=\left(t_{0}, t_{1}\right)$ and $[z] \in W$ being a point determined by a matrix $z$. Note that any element $[z] \in W$ determines a projective line $\Phi\left(\mathbb{P}^{1} \times\{[z]\}\right)$ in the twistor space $\mathbb{P}^{N}$. The space $\mathrm{Gr}_{2, N+1}$ is called the space-time on which solutions of $\mathfrak{g l}_{r}(\mathbb{C})$ GASDYM are defined, and $\mathbb{P}^{N}$ is called the twistor space on which the corresponding holomorphic vector bundles of rank $r$, trivial on the projective lines, are defined by Ward transform.

To state the main theorem, we shall define the action of $H_{\lambda}$ on $\mathbb{P}^{N}$. Let $x=\left(x_{0}, x_{1}, \ldots, x_{N}\right)$ be the homogeneous coordinates of $\mathbb{P}^{N}$ and $[x]$ denote the point of $\mathbb{P}^{N}$ with the homogeneous coordinates $x$. Define the right action of $H_{\lambda}$ on $\mathbb{P}^{N}$ by

$$
\mathbb{P}^{N} \times H_{\lambda} \rightarrow \mathbb{P}^{N}, \quad([x], h) \mapsto[x h] .
$$

If we write the homogeneous coordinate $x$ in blocks as

$$
x=\left(x^{(1)}, \ldots, x^{(\ell)}\right), \quad x^{(k)}=\left(x_{0}^{(k)}, \ldots, x_{n_{k}-1}^{(k)}\right)
$$

according as the partition $\lambda=\left(n_{1}, \ldots, n_{\ell}\right)$, then the action of $h=\left(h^{(1)}, \ldots, h^{(\ell)}\right) \in H_{\lambda}$ is written as

$$
[x h]=\left[x^{(1)} h^{(1)}, \ldots, x^{(\ell)} h^{(\ell)}\right] .
$$

Theorem 3.1. (Mason and Woodhouse [6]) Let $U \subset \mathbb{P}^{N}$ be an open set and $\pi: E \rightarrow U$ be a rank $r$ holomorphic vector bundle. Assume that the following conditions are fulfilled.

1. $U$ is invariant by the action of $H_{\lambda}$, namely, for $[x] \in U$ and $h \in H_{\lambda}$, we have $[x h] \in U$, 
2. the action of $H_{\lambda}$ on $U$ can be analytically lifted to $E$.

Then the infinitesimal action of $H_{\lambda}$ to $E$ defines a flat connection $\nabla_{\lambda}$ on $E$.

The explicit form of the connection $\nabla_{\lambda}$ is given as follows.

Proposition 3.1. The flat connection $\nabla_{\lambda}$ in Theorem 3.1 can be written locally as

$$
\nabla_{\lambda}=d-\left(\sum_{k=1}^{\ell} \sum_{\alpha=0}^{n_{k}-1} A_{\alpha}^{(k)}(x) d \theta_{\alpha}\left(x^{(k)}\right)\right) \wedge,
$$

where $A_{j}^{(k)}(x) \in \mathfrak{g l}_{r}(\mathbb{C})$ are holomorphic in an open subset $V \subset U$ on which the bundle $E$ is trivial.

In order to relate this flat connection to the isomonodromic deformation of an ordinary differential equation on $\mathbb{P}^{1}$, we use the picture (3.1) replacing the subset $W \subset \mathrm{Gr}_{2, N+1}$ with the open subset $Z_{\lambda} \subset Z$, defined below, whose image is $W$ by the map $Z \rightarrow \mathrm{Gr}_{2, N+1}=\mathrm{GL}_{2}(\mathbb{C}) \backslash Z$. The coordinates of $Z_{\lambda}$ will play an analogous role for $W$ to the homogeneous coordinates for a projective space and will be convenient for describing the systems we are going to treat in a symmetric manner.

Given a matrix $z \in Z$, we write $z$ as

$$
z=\left(z^{(1)}, \ldots, z^{(\ell)}\right), \quad z^{(k)}=\left(z_{0}^{(k)}, \ldots, z_{n_{k}-1}^{(k)}\right) \in \operatorname{Mat}_{2, n_{k}}(\mathbb{C}),
$$

where $z_{i}^{(k)}$ is a two dimensional column vector. Define an open subset $Z_{\lambda}$ of $\operatorname{Mat}_{2, N+1}(\mathbb{C})$ by

$$
Z_{\lambda}=\left\{z \in Z \mid \begin{array}{lr}
\operatorname{det}\left(z_{0}^{(k)}, z_{1}^{(k)}\right) \neq 0 & \left(n_{k} \geq 2\right) \\
\operatorname{det}\left(z_{0}^{(k)}, z_{0}^{(l)}\right) \neq 0 & (k \neq l)
\end{array}\right\} .
$$

It is seen that the map $Z \times H_{\lambda} \ni(z, h) \mapsto z h \in Z$ defines an action of $H_{\lambda}$ on $Z_{\lambda}$. Now the map $\Phi: \mathbb{P}^{1} \times Z_{\lambda} \rightarrow \mathbb{P}^{N}$ is the holomorphic map defined by

$$
([\vec{\zeta}], z) \mapsto[\vec{\zeta} z]=\left[\vec{\zeta} z^{(1)}, \ldots, \vec{\zeta} z^{(\ell)}\right],
$$

where $\vec{\zeta}=(1, \zeta)$ and $\zeta$ denotes the affine coordinate of $\mathbb{P}^{1}$. Then we can relate the connection $\nabla_{\lambda}$ to the isomonodromic deformation in explicit manner.

Theorem 3.2. Let $U \subset \mathbb{P}^{N}$ be an open set containing a projective line and let $\pi: E \rightarrow U$ be a holomorphic vector bundle on $U$ of rank $r$. Assume that

1. $U$ is invariant by the action of $H_{\lambda}$ on $\mathbb{P}^{N}$ defined by (3.2),

2. $E$ is trivial on projective lines contained in $U$,

3. the action of $H_{\lambda}$ on $U$ can be lifted to $E$.

Then the flat connection $\tilde{\nabla}_{\lambda}=\Phi^{*} \nabla_{\lambda}$ on $\Phi^{*} E$ obtained from $\nabla_{\lambda}$ in Theorem 3.1 gives the isomonodromic deformation of a system of linear differential equation

$$
\frac{d y}{d \zeta}=\left(\sum_{k=1}^{\ell} \sum_{\alpha=0}^{n_{k}-1} \tilde{A}_{\alpha}^{(k)}(z) \frac{d \theta_{\alpha}\left(\vec{\zeta}_{z}^{(k)}\right)}{d \zeta}\right) y
$$

with an unknown vector $y \in \mathbb{C}^{r}$.

\section{Remark 3.1.}




\section{H. Kimura and D. Tseveenamijil}

1. This result is already announced in [5] without explicit form of connections $\tilde{\nabla}_{\lambda}$.

2. $\tilde{A}_{\alpha}^{(k)}(z)$ depends on $z$ but not on $\zeta$ as is explained in the proof.

3. $d \theta_{j}\left(\vec{\zeta} z^{(k)}\right) / d \zeta$, as a function of $\zeta$, has a pole $\zeta=-z_{00}^{(k)} / z_{10}^{(k)}$ of order $j+1$.

4. By the action (3.2) of $H_{\lambda}, \mathbb{P}^{N}$ is expressed as a union of orbits. There is an open dense orbit $O(a)$ passing through $[a] \in \mathbb{P}^{N}$, where

$$
a=\left(a^{(1)}, \ldots, a^{(\ell)}\right) \in \mathbb{C}^{N+1}, \quad a^{(k)}=(1,0, \ldots, 0) \in \mathbb{C}^{n_{k}}
$$

and there are codimension 1 orbits $O\left(b_{j}\right),(j=1, \ldots, \ell)$, where $b_{j}=\left(b_{j}^{(1)}, \ldots, b_{j}^{(\ell)}\right)$ with

$$
b_{j}^{(k)}=(1,0, \ldots, 0), \quad(k \neq j), \quad b_{j}^{(j)}=(0,1,0, \ldots, 0) .
$$

When $U=O(a) \cup O\left(b_{1}\right) \cup \cdots \cup O\left(b_{\ell}\right)$, the set $Z_{\lambda}$ is the space which parametrizes all the projective lines contained in $U$.

Proposition 3.2. The flat connection $\tilde{\nabla}_{\lambda}$ in Theorem 3.2 is locally written as

$$
\tilde{\nabla}_{\lambda}=d-\left(\sum_{k=1}^{\ell} \sum_{\alpha=0}^{n_{k}-1} \tilde{A}_{\alpha}^{(k)}(z) d \theta_{\alpha}\left(\vec{\zeta}_{z}\right)\right) \wedge .
$$

Definition 3.1. The nonlinear system of differential equations for $\tilde{A}_{\alpha}^{(k)}$ obtained as the complete integrability condition of (3.8) is called the general Schlesinger system (GSS) of type $\lambda$.

\section{Proof of Main Results}

\subsection{Proof of Proposition 3.1}

We fix a partition $\lambda$ of $N+1$ and denote $H_{\lambda}$ and $\mathfrak{h}_{\lambda}$ by $H$ and $\mathfrak{h}$, respectively. Let $[a] \in \mathbb{P}^{N}$ be the point defined by $a \in \mathbb{C}^{N+1}$ given in (3.7). Define the map $\varphi: \mathfrak{h} \rightarrow \mathbb{P}^{N}$ by

$$
\varphi(\xi)=\left[a \cdot e^{\xi}\right]
$$

The image $\varphi(\mathfrak{h})$ coincides with an open dense orbit $O(a)$ in $\mathbb{P}^{N}$ by the action (3.2). Let $\xi \in \mathfrak{h}$ and put

$$
\xi=\left(\xi^{(1)}, \ldots, \xi^{(\ell)}\right), \quad \xi^{(k)}=\left[\xi_{0}^{(k)}, \xi_{1}^{(k)}, \ldots, \xi_{n_{k}-1}^{(k)}\right] \in \mathfrak{j}\left(n_{k}\right)
$$

Take the commuting vector fields $\partial / \partial \xi_{\alpha}^{(k)}\left(1 \leq k \leq \ell ; 0 \leq \alpha<n_{k}\right)$ on $\mathfrak{h}$ and let

$$
X_{\alpha}^{(k)}=\varphi_{*} \frac{\partial}{\partial \xi_{\alpha}^{(k)}} .
$$

be the vector fields on $O(a)$ obtained by $\varphi_{*}:\left.T \mathfrak{h} \rightarrow T \mathbb{P}^{N}\right|_{O(a)}$. Note that $X_{\alpha}^{(k)}$ 's span the tangent space $T_{[x]} \mathbb{P}^{N}$ at any point $[x] \in O(a)$.

Lemma 4.1. The vector field $X_{\alpha}^{(k)}$ has the form

$$
X_{\alpha}^{(k)}=x_{0}^{(k)} \frac{\partial}{\partial x_{\alpha}^{(k)}}+x_{1}^{(k)} \frac{\partial}{\partial x_{\alpha+1}^{(k)}}+\cdots+x_{n_{k}-\alpha-1}^{(k)} \frac{\partial}{\partial x_{n_{k}-1}^{(k)}} .
$$


Proof. The map $\varphi$ is induced from the map

$$
\mathfrak{h} \ni \xi=\left(\xi^{(1)}, \ldots, \xi^{(\ell)}\right) \mapsto x(\xi)=\left(a^{(1)} e^{\xi^{(1)}}, \ldots, a^{(\ell)} e^{\xi^{(\ell)}}\right) \in \mathbb{C}^{N+1} .
$$

Since $x^{(k)}$ depends only on $\xi^{(k)}$, the vector field $X_{\alpha}^{(k)}$ is written in terms of $x^{(k)}$. To compute explicitly $X_{\alpha}^{(k)}$ from (4.1), it is sufficient to consider only $\xi^{(k)}, x^{(k)}$. To simplify notations, we write $n$ instead of $n_{k}$ and $x=\left(x_{0}, \ldots, x_{n-1}\right), \xi=\left(\xi_{0}, \ldots, \xi_{n-1}\right), X_{\alpha}$, instead of $x^{(k)}, \xi^{(k)}, X_{\alpha}^{(k)}$, respectively, removing the suffix $(k)$. Then

$$
\left(x_{0}, x_{1}, \ldots, x_{n-1}\right)=\left(e^{\xi_{0}} p_{0}(\xi), e^{\xi_{0}} p_{1}(\xi), \ldots, e^{\xi_{0}} p_{n-1}(\xi)\right)
$$

and we have

$$
\begin{aligned}
X_{\alpha} & =\frac{\partial x_{0}}{\partial \xi_{\alpha}} \frac{\partial}{\partial x_{0}}+\frac{\partial x_{1}}{\partial \xi_{\alpha}} \frac{\partial}{\partial x_{1}}+\cdots+\frac{\partial x_{n-1}}{\partial \xi_{\alpha}} \frac{\partial}{\partial x_{n-1}} \\
& =e^{\xi_{0}} p_{0}(\xi) \frac{\partial}{\partial x_{\alpha}}+e^{\xi_{0}} p_{1}(\xi) \frac{\partial}{\partial x_{\alpha+1}}+\cdots+e^{\xi_{0}} p_{n-1-\alpha}(\xi) \frac{\partial}{\partial x_{n-1}} \\
& =x_{0} \frac{\partial}{\partial x_{\alpha}}+x_{1} \frac{\partial}{\partial x_{\alpha+1}}+\cdots+x_{n-1-\alpha} \frac{\partial}{\partial x_{n-1}}
\end{aligned}
$$

Here we used the the identity

$$
\frac{\partial p_{j}}{\partial \xi_{\alpha}}=p_{j-\alpha} \quad(j \geq \alpha)
$$

which can be obtained by differentiating the identity (2.6).

For a general partition $\lambda=\left(n_{1}, \ldots, n_{\ell}\right)$ of $N+1$, we thus obtained the commuting vector fields on $\mathbb{P}^{N}$

$$
X_{0}^{(1)}, \ldots, X_{n_{1}-1}^{(1)}, \ldots, X_{0}^{(\ell)}, \ldots, X_{n_{\ell}-1}^{(\ell)}
$$

Remark 4.1. The vector field $X_{0}^{(1)}+\cdots+X_{0}^{(\ell)}$ on $\mathbb{C}^{N+1}$ is an Euler vector field and reduces to a zero vector field on $\mathbb{P}^{N}$.

By the second assumption of Theorem 3.1, we can lift $X_{\alpha}^{(k)}$ to a vector field $\tilde{X}_{\alpha}^{(k)}$ on $E$. Since $\pi_{*} \tilde{X}_{\alpha}^{(k)}=X_{\alpha}^{(k)}$, we can put

$$
\tilde{X}_{\alpha}^{(k)}=X_{\alpha}^{(k)}+Y_{\alpha}^{(k)}
$$

where $Y_{\alpha}^{(k)}$ is a vector field tangent to the fibers of $E$. If we take a trivialization of $\pi^{-1}(V) \simeq V \times \mathbb{C}^{r}$ with coordinates $(x, y) \in V \times \mathbb{C}^{r}$ for some $V \subset U$, the vector field $Y_{\alpha}^{(k)}$ is expressed as

$$
Y_{\alpha}^{(k)}=\sum_{i, j} y_{j} A_{\alpha i j}^{(k)}(x) \frac{\partial}{\partial y_{i}}, \quad A_{\alpha i j}^{(k)}(x) \in \mathscr{O}(V) .
$$

Since $\tilde{X}_{\alpha}^{(k)}$ are commuting vector fields by definition, they determine a completely integrable distribution $\mathscr{D}$ which corresponds to a flat connection on the bundle $E$. Put

$$
A_{\alpha}^{(k)}(x)=\left(A_{\alpha i j}^{(k)}(x)\right)_{0 \leq i, j<r} \in \mathfrak{g l}_{r}(\mathbb{C}) .
$$


Remark 4.2. The integrability of the distribution $\mathscr{D}$ is written locally as

$$
X_{\alpha}^{(k)} A_{\beta}^{\left(k^{\prime}\right)}-X_{\beta}^{\left(k^{\prime}\right)} A_{\alpha}^{(k)}-\left[A_{\alpha}^{(k)}, A_{\beta}^{\left(k^{\prime}\right)}\right]=0
$$

Let $\nabla$ be the flat connection corresponding to $\mathscr{D}$ whose locally constant sections are the leaves of the foliation determined by $\mathscr{D}$. Write the connection $\nabla$ as

$$
\nabla=d-\omega \wedge
$$

where the connection form $\omega$ is expressed in a local trivialization $V \times \mathbb{C}^{r}$ as

$$
\omega=\sum_{k=1}^{\ell} \omega^{(k)}, \quad \omega^{(k)}=\sum_{\alpha=0}^{n_{k}-1} B_{\alpha}^{(k)}(x) d x_{\alpha}^{(k)}, \quad B_{\alpha}^{(k)} \in \mathfrak{g l}_{r}(\mathbb{C})
$$

Let us express $B_{\alpha}^{(k)}$ in terms of $A_{\alpha}^{(k)}$, s.

Lemma 4.2. For $0 \leq \alpha<n_{k}$, we have

$$
x_{0}^{(k)} B_{\alpha}^{(k)}+x_{1}^{(k)} B_{\alpha+1}^{(k)}+\cdots+x_{n_{k}-1-\alpha}^{(k)} B_{n_{k}-1}^{(k)}=A_{\alpha}^{(k)} .
$$

Proof. Let $x \mapsto(x, y(x))$ be a locally constant section of $\nabla$ in the local trivialization. We compute the tangent vector to this section. Since the orbit passing through $[x] \in \mathbb{P}^{N}$ is expressed as

$$
\xi \mapsto x(\xi)=x e^{\xi}
$$

and the tangent vector $X_{\alpha}^{(k)}$ is the directional derivation corresponding to $\partial / \partial \xi_{\alpha}^{(k)}$, we have

$$
\begin{aligned}
X_{\alpha}^{(k)} y & =\left.\frac{\partial}{\partial \xi_{\alpha}^{(k)}} y\left(x e^{\xi}\right)\right|_{\xi=0}=\left.\sum_{\beta} \frac{\partial x_{\beta}^{(k)}(\xi)}{\partial \xi_{\alpha}^{(k)}} \frac{\partial}{\partial x_{\beta}^{(k)}} y\right|_{\xi=0} \\
& =\sum_{\beta} x_{\beta-\alpha}^{(k)} \frac{\partial}{\partial x_{\beta}^{(k)}} y=\left(\sum_{\beta \geq \alpha} x_{\beta-\alpha}^{(k)} B_{\beta}^{(k)}\right) y .
\end{aligned}
$$

Here we used the fact that $y(x)$ is a locally constant section of $\nabla$, namely $\nabla y=0$. Thus we must have $A_{\alpha}^{(k)}=\sum_{\beta} x_{\beta-\alpha}^{(k)} B_{\beta}^{(k)}$, which gives (4.5).

Definition 4.1. The rational functions $\phi_{m}(x)$ of $x=\left(x_{0}, x_{1}, \ldots\right)$ are defined by the generating function

$$
\left(x_{0}+x_{1} T+x_{2} T^{2}+\cdots\right)^{-1}=\sum_{m=0}^{\infty} \phi_{m}(x) T^{m}
$$


A few of $\phi_{m}(x)$ are written as follows.

$$
\begin{aligned}
& \phi_{0}(x)=\frac{1}{x_{0}} \\
& \phi_{1}(x)=\frac{1}{x_{0}}\left(-\frac{x_{1}}{x_{0}}\right), \\
& \phi_{2}(x)=\frac{1}{x_{0}}\left\{-\frac{x_{2}}{x_{0}}+\left(\frac{x_{1}}{x_{0}}\right)^{2}\right\}, \\
& \phi_{3}(x)=\frac{1}{x_{0}}\left\{-\frac{x_{3}}{x_{0}}+2\left(\frac{x_{1}}{x_{0}}\right)\left(\frac{x_{2}}{x_{0}}\right)-\left(\frac{x_{1}}{x_{0}}\right)^{3}\right\}, \\
& \phi_{4}(x)=\frac{1}{x_{0}}\left\{-\frac{x_{4}}{x_{0}}+\left(\frac{x_{2}}{x_{0}}\right)^{2}+2\left(\frac{x_{1}}{x_{0}}\right)\left(\frac{x_{3}}{x_{0}}\right)-3\left(\frac{x_{1}}{x_{0}}\right)^{2}\left(\frac{x_{2}}{x_{0}}\right)+\left(\frac{x_{1}}{x_{0}}\right)^{4}\right\} .
\end{aligned}
$$

The relation between the functions $\theta_{m}$ and $\phi_{m}$ is given by the following lemma, which can be shown by differentiating the both side of (2.4) with respect to $x_{\alpha}$.

\section{Lemma 4.3.}

$$
\frac{\partial \theta_{m}}{\partial x_{\alpha}}=\phi_{m-\alpha}
$$

Now we shall complete the proof of Proposition 3.1. As in the proof of Lemma 4.1, we omit a superfix $(k)$ and a suffix $k$ from the relation (4.5) for the sake of simplicity of notation. The relation (4.5) can be written symbolically as

$$
\left(\begin{array}{c}
A_{0} \\
\vdots \\
A_{n-2} \\
A_{n-1}
\end{array}\right)=\left(\begin{array}{cccc}
x_{0} & x_{1} & \ldots & x_{n-1} \\
& \ddots & \ddots & \vdots \\
& & \ddots & x_{1} \\
& & & x_{0}
\end{array}\right)\left(\begin{array}{c}
B_{0} \\
\vdots \\
B_{n-2} \\
B_{n-1}
\end{array}\right)
$$

Solving it with respect to $B_{i}$ 's we have

$$
\begin{aligned}
\left(\begin{array}{c}
B_{0} \\
\vdots \\
B_{n-2} \\
B_{n-1}
\end{array}\right)= & \left(\begin{array}{cccc}
x_{0} & x_{1} & \ldots & x_{n-1} \\
& \ddots & \ddots & \vdots \\
& & \ddots & x_{1} \\
& & & x_{0}
\end{array}\right)^{-1}\left(\begin{array}{c}
A_{0} \\
\vdots \\
A_{n-2} \\
A_{n-1}
\end{array}\right) \\
= & \left(\begin{array}{cccc}
\phi_{0} & \phi_{1} & \ldots & \phi_{n-1} \\
& \ddots & \ddots & \vdots \\
& & \ddots & \phi_{1} \\
& & \phi_{0}
\end{array}\right)\left(\begin{array}{c}
A_{0} \\
\vdots \\
A_{n-2} \\
A_{n-1}
\end{array}\right) .
\end{aligned}
$$

Here we used the rational functions $\phi_{m}(x)$ introduced in Def. 4.1.

To complete the proof of Pro. 3.1, it is sufficient to show the following. 
Lemma 4.4. We have

$$
\omega^{(k)}=\sum_{0 \leq \alpha<n_{k}} A_{\alpha}^{(k)} d \theta_{\alpha}\left(x^{(k)}\right) .
$$

Proof. Adopting the above notation we have

$$
\begin{aligned}
& \omega^{(k)}=\sum_{\alpha} B_{\alpha} d x_{\alpha} \\
& =\left(d x_{0}, \ldots, d x_{n-1}\right)\left(\begin{array}{c}
B_{0} \\
\vdots \\
B_{n-2} \\
B_{n-1}
\end{array}\right) \\
& =\left(d x_{0}, \ldots, d x_{n-1}\right)\left(\begin{array}{cccc}
\phi_{0} & \phi_{1} & \ldots & \phi_{n-1} \\
& \ddots & \ddots & \vdots \\
& & \ddots & \phi_{1} \\
& & \phi_{0}
\end{array}\right)\left(\begin{array}{c}
A_{0} \\
\vdots \\
A_{n-2} \\
A_{n-1}
\end{array}\right) \\
& =\left(\phi_{0} d x_{0}, \phi_{1} d x_{0}+\phi_{0} d x_{1}, \ldots, \phi_{n-1} d x_{0}+\cdots+\phi_{0} d x_{n-1}\right)\left(\begin{array}{c}
A_{0} \\
\vdots \\
A_{n-2} \\
A_{n-1}
\end{array}\right) \\
& =\left(d \theta_{0}, d \theta_{1}, \ldots, d \theta_{n-1}\right)\left(\begin{array}{c}
A_{0} \\
\vdots \\
A_{n-2} \\
A_{n-1}
\end{array}\right) .
\end{aligned}
$$

Here we used the relation

$$
\phi_{m} d x_{0}+\phi_{m-1} d x_{1}+\cdots+\phi_{0} d x_{m}=d \theta_{m},
$$

which can be derived by taking the exterior derivative of $\theta_{m}$ and using Lemma 4.3. This proves the lemma.

\subsection{Proof of Theorem 3.2}

Let $\nabla_{\lambda}$ be that given by (3.4). Let $L$ be a projective line contained in $U \subset \mathbb{P}^{N}$ determined by $z \in$ $\operatorname{Mat}_{2, N+1}(\mathbb{C})$ as

$$
\mathbb{P}^{1} \ni[\vec{\zeta}] \mapsto[\vec{\zeta} z] \in \mathbb{P}^{N}
$$

Since the bundle $E$ is trivial on $L$ by the assumption, the connection $\Phi^{*} \nabla_{\lambda}$ is globally written as

$$
\Phi^{*} \nabla_{\lambda}=d-\left(\sum_{k=1}^{\ell} \sum_{\alpha=0}^{n_{k}-1} A_{\alpha}^{(k)}\left(\vec{\zeta}_{z}\right) d \theta_{\alpha}\left(\vec{\zeta}_{z}\right)\right) \wedge
$$


where $A_{\alpha}^{(k)}(\vec{\zeta} z)$ is holomorphic on $L\left(\simeq \mathbb{P}^{1}\right)$. By Liouville Theorem, we see that $A_{\alpha}^{(k)}(\vec{\zeta} z)$ does not depend on $\zeta$, which we denote as $\tilde{A}_{\alpha}^{(k)}(z)$.

\section{Examples}

\subsection{Schlesinger system}

We show that the Schlesinger system, which describes the isomonodromic deformation of the Fuchsian system

$$
\frac{d y}{d x}=\left(\sum_{j=0}^{N} \frac{\tilde{A}_{j}(t)}{x-t_{j}}\right) y . \quad \sum_{j=0}^{N} \tilde{A}_{j}(t)=0
$$

with additional condition on the eigenvalues of $\tilde{A}_{j}(t)$, can be derived from Theorem 3.2. Take a partition $\lambda=(1, \ldots, 1)$ of $N+1$ and take the subset

$$
X_{\lambda}=\left\{z=\left(\begin{array}{cccc}
-t_{0} & -t_{1} & \ldots & -t_{N} \\
1 & 1 & \ldots & 1
\end{array}\right) \mid t_{i} \neq t_{j} \quad(i \neq j)\right\} \subset Z_{\lambda}
$$

The group $H_{\lambda}$ in this case is the Cartan subgroup of $\mathrm{GL}_{N+1}(\mathbb{C})$ consisting of diagonal matrices. Then $X_{\lambda}$ is a realization of the quotient space $\left(Z_{\lambda} \cap\left\{z_{10} \cdots z_{1 N} \neq 0\right\}\right) / H_{\lambda}$. Define the map $\Phi$ : $\mathbb{P}^{1} \times X_{\lambda} \rightarrow \mathbb{P}^{N}$ by

$$
([\vec{\zeta}], z) \mapsto[\vec{\zeta} z]=\left[\zeta-t_{0}, \ldots, \zeta-t_{N}\right] .
$$

The pull back $\Phi^{*} \nabla=d-\Phi^{*} \omega \wedge$ of the flat connection $\nabla$ in Theorem 3.2 is written locally as

$$
\Phi^{*} \omega=\sum_{j=0}^{N} \tilde{A}_{j}(t) d \log \left(\zeta-t_{j}\right), \quad \sum_{j} \tilde{A}_{j}(t)=0,
$$

where $\tilde{A}_{j}(t)$ are $r \times r$ matrix functions depending on $t$. Then the flatness condition $\Phi^{*}(d \omega-\omega \wedge$ $\omega)=0$ of $\Phi^{*} \nabla$ is the Schlesinger system

$$
d \tilde{A}_{j}+\sum_{i \neq j}\left[\tilde{A}_{j}, \tilde{A}_{i}\right] \frac{d t_{i}-d t_{j}}{t_{i}-t_{j}}=0, \quad(0 \leq j \leq N) .
$$

\subsection{Painlevé equations}

The Painleve equations can be obtained in the context of Theorem 3.2, where the bundle $E$ is of rank 2 and the partition $\lambda$ is of weight 4 . We list up, for each Painlevé equation, the following data:

1. a partition $\lambda$ and the abelian group $H_{\lambda}$,

2. the subspace $X_{\lambda}$ of $Z_{\lambda}$ which is a realization of $\mathrm{GL}_{2}(\mathbb{C}) \backslash Z_{\lambda} / H_{\lambda}$ and parametrizes lines in an invariant open subset $U$ of $\mathbb{P}^{3}$,

3. the map $\Phi: \mathbb{P}^{1} \times X_{\lambda} \rightarrow \mathbb{P}^{3}$,

4. the form of flat connection $\nabla$,

5. the connection form $\Phi^{*} \omega$ of $\Phi^{*} \nabla$ obtained by the pull back,

6. the system of linear differential equations describing the isomonodromic deformation,

7. the system of nonlinear equations equivalent to the Painlevé equation (see [7,8] for the equivalence). 
We use the convention to write $A_{\alpha}$ for $\tilde{A}_{\alpha}$ in the connection form $\Phi^{*} \omega$. Note that in each of the following cases, the invariant open subset $U \subset \mathbb{P}^{3}$ in Theorem 3.2 is a union of the open dense orbit and the codimension 1 orbits.

\subsubsection{Painlevé $P_{V I}$}

1. $\lambda=(1,1,1,1), \quad H_{\lambda}=\left\{\left(\begin{array}{llll}h_{0} & & & \\ & h_{1} & & \\ & & h_{2} & \\ & & & h_{3}\end{array}\right)\right\}$.

2. $X_{\lambda}=\left\{z=\left(\begin{array}{cccc}1 & 0 & -1 & -t \\ 0 & 1 & 1 & 1\end{array}\right) \mid t \neq 0,1, \infty\right\}:$ a realization of the configuration space of 4 points of $\mathbb{P}^{1}$ in general position.

3. $\Phi: \mathbb{P}^{1} \times X_{\lambda} \rightarrow \mathbb{P}^{3},(\zeta, z) \mapsto[1, \zeta, \zeta-1, \zeta-t]$.

4. $\nabla=d-\left(A_{0} d \log x_{0}+A_{1} d \log x_{1}+A_{2} d \log x_{2}+A_{3} d \log x_{3}\right) \wedge$, with $A_{0}+A_{1}+A_{2}+A_{3}=0$.

5. $\Phi^{*} \omega=A_{1} \frac{d \zeta}{\zeta}+A_{2} \frac{d \zeta}{\zeta-1}+A_{3} \frac{d \zeta-d t}{\zeta-t}$.

6. $\Phi^{*} \nabla y=0 \Leftrightarrow$

$$
\left\{\begin{array}{l}
\frac{\partial y}{\partial \zeta}=\left(\frac{A_{1}}{\zeta}+\frac{A_{2}}{\zeta-1}+\frac{A_{3}}{\zeta-t}\right) y \\
\frac{\partial y}{\partial t}=-\frac{A_{3}}{\zeta-t} y
\end{array}\right.
$$

7. $\Phi^{*} \nabla^{2}=0 \Leftrightarrow$ the system equivalent to $P_{V I}($ see $(1.3))$ :

$$
\frac{d A_{1}}{d t}=\frac{\left[A_{3}, A_{1}\right]}{t}, \quad \frac{d A_{2}}{d t}=\frac{\left[A_{3}, A_{2}\right]}{t-1}, \quad \frac{d A_{3}}{d t}=-\frac{\left[A_{3}, A_{1}\right]}{t}-\frac{\left[A_{3}, A_{2}\right]}{t-1} .
$$

\subsubsection{Painlevé $P_{V}$}

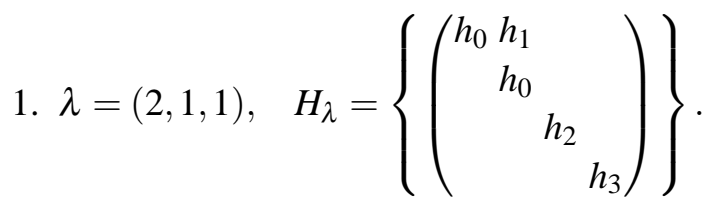

2. $X_{\lambda}=\left\{z=\left(\begin{array}{cccc}1 & 0 & 0 & -t \\ 0 & 1 & 1 & 1\end{array}\right) \mid t \neq 0, \infty\right\}$.

3. $\Phi: \mathbb{P}^{1} \times X_{\lambda} \rightarrow \mathbb{P}^{3}:(\zeta, z) \mapsto[1, \zeta, \zeta, \zeta-t]$.

4. $\nabla=d-\left(A_{0} d \log x_{0}+A_{1} d \theta_{1}+A_{2} d \log x_{2}+A_{3} d \log x_{3}\right) \wedge \quad$, with $A_{0}+A_{2}+A_{3}=0$.

5. $\Phi^{*} \omega=A_{1} d \zeta+A_{2} \frac{d \zeta}{\zeta}+A_{3} \frac{d \zeta-d t}{\zeta-t}$.

6. $\Phi^{*} \nabla y=0 \Leftrightarrow$

$$
\left\{\begin{array}{l}
\frac{\partial y}{\partial \zeta}=\left(A_{1}+\frac{A_{2}}{\zeta}+\frac{A_{3}}{\zeta-t}\right) y \\
\frac{\partial y}{\partial t}=-\frac{A_{3}}{\zeta-t} y
\end{array}\right.
$$


7. $\Phi^{*} \nabla^{2}=0 \Leftrightarrow$ the system equivalent to $P_{V}$ :

$$
\frac{d A_{1}}{d t}=0, \quad \frac{d A_{2}}{d t}=\frac{\left[A_{3}, A_{2}\right]}{t}, \quad \frac{d A_{3}}{d t}=\left[A_{1}, A_{3}\right]-\frac{\left[A_{3}, A_{2}\right]}{t} .
$$

\subsubsection{Painlevé $P_{I V}$}

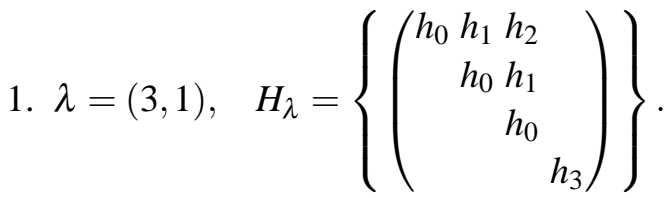
2. $X_{\lambda}=\left\{z=\left(\begin{array}{cccc}1 & 0 & 0 & -t \\ 0 & 1 & 0 & 1\end{array}\right) \mid t \neq \infty\right\}$.
3. $\Phi: \mathbb{P}^{1} \times X_{\lambda} \rightarrow \mathbb{P}^{3},(\zeta, z) \mapsto[1, \zeta, 0, \zeta-t]$.
4. $\nabla=d-\left(A_{0} d \log x_{0}+A_{1} d \theta_{1}+A_{2} d \theta_{2}+A_{3} d \log x_{3}\right) \wedge \quad$, with $A_{0}+A_{3}=0$.
5. $\Phi^{*} \omega=A_{1} d \zeta-A_{2} \zeta d \zeta+A_{3} \frac{d \zeta-d t}{\zeta-t}$.
6. $\Phi^{*} \nabla y=0 \Leftrightarrow$

$$
\left\{\begin{array}{l}
\frac{\partial y}{\partial \zeta}=\left(A_{1}-\zeta A_{2}+\frac{A_{3}}{\zeta-t}\right) y \\
\frac{\partial y}{\partial t}=-\frac{A_{3}}{\zeta-t} y
\end{array}\right.
$$

7. $\Phi^{*} \nabla^{2}=0 \Leftrightarrow$ the system equivalent to $P_{I V}$ :

$$
\frac{d A_{1}}{d t}=\left[A_{3}, A_{2}\right], \quad \frac{d A_{2}}{d t}=0, \quad \frac{d A_{3}}{d t}=\left[A_{1}-t A_{2}, A_{3}\right] .
$$

\subsubsection{Painlevé $P_{I I I}$}

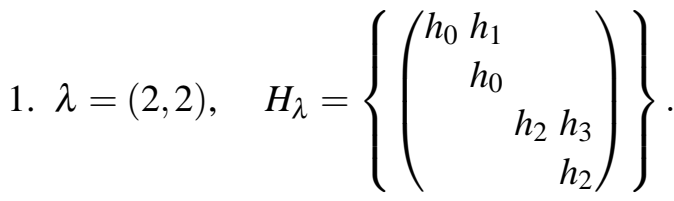

2. $X_{\lambda}=\left\{z=\left(\begin{array}{llll}1 & 0 & 0 & t \\ 0 & 1 & 0 & 0\end{array}\right) \mid t \neq 0, \infty\right\}$.

3. $\Phi: \mathbb{P}^{1} \times X_{\lambda} \rightarrow \mathbb{P}^{3},(\zeta, z) \mapsto[1, \zeta, \zeta, t]$.

4. $\nabla=d-\left(A_{0} d \log x_{0}+A_{1} d \theta_{1}+A_{2} d \log x_{2}+A_{3} d \theta_{1}\left(x_{2}, x_{3}\right)\right) \wedge \quad$, with $A_{0}+A_{2}=0$.

5. $\Phi^{*} \omega=A_{1} d \zeta+A_{2} \frac{d \zeta}{\zeta}+A_{3} d\left(\frac{t}{\zeta}\right)$

6. $\Phi^{*} \nabla y=0 \Leftrightarrow$

$$
\left\{\begin{array}{l}
\frac{\partial y}{\partial \zeta}=\left(A_{1}+\frac{A_{2}}{\zeta}-\frac{t A_{3}}{\zeta^{2}}\right) y \\
\frac{\partial y}{\partial t}=\frac{A_{3}}{\zeta} y
\end{array}\right.
$$


7. $\Phi^{*} \nabla^{2}=0 \Leftrightarrow$ the system equivalent to $P_{I I I}$ :

$$
\frac{d A_{1}}{d t}=0, \quad \frac{d A_{2}}{d t}=\left[A_{3}, A_{1}\right], \quad \frac{d A_{3}}{d t}=\frac{\left[A_{2}, A_{3}\right]}{t} .
$$

\subsubsection{Painlevé $P_{I I}$}

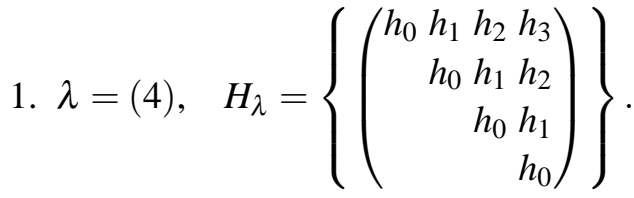

2. $X_{\lambda}=\left\{z=\left(\begin{array}{llll}1 & 0 & t & 0 \\ 0 & 1 & 0 & 0\end{array}\right) \mid t \neq \infty\right\}$.

3. $\Phi: \mathbb{P}^{1} \times X_{\lambda} \rightarrow \mathbb{P}^{3},(\zeta, z) \mapsto[1, \zeta, t, 0]$.

4. $\nabla=d-\left(A_{0} d \log x_{0}+A_{1} d \theta_{1}+A_{2} d \theta_{2}+A_{3} d \theta_{3}\right) \wedge$, with $A_{0}=0$.

5. $\Phi^{*} \omega=A_{1} d \zeta+A_{2}(d t-\zeta d \zeta)+A_{3}\left(-d(\zeta t)+\zeta^{2} d \zeta\right)$.

6. $\Phi^{*} \nabla y=0 \Leftrightarrow$

$$
\left\{\begin{array}{l}
\frac{\partial y}{\partial \zeta}=\left(A_{1}-\zeta A_{2}+\left(-t+\zeta^{2}\right) A_{3}\right) y \\
\frac{\partial y}{\partial t}=\left(A_{2}-\zeta A_{3}\right) y
\end{array}\right.
$$

7. $\Phi^{*} \nabla^{2}=0 \Leftrightarrow$ the system equivalent to $P_{I I}$ :

$$
\frac{d A_{1}}{d t}=\left[A_{2}, A_{1}-t A_{3}\right], \quad \frac{d A_{2}}{d t}=\left[A_{3}, A_{1}\right], \quad \frac{d A_{3}}{d t}=0 .
$$

\section{Symmetry}

When $H$ is a Cartan subgroup of $G=\mathrm{GL}_{N+1}(\mathbb{C})$, we have the Weyl group $W=N_{G}(H) / H$, where $N_{G}(H)$ is the normalizer of $H$, which is isomorphic to the symmetric group $\mathfrak{S}_{N+1}$. Here we consider an analogue of the Weyl group replacing $H$ with the abelian group $H_{\lambda}$. In the theory of hypergeometric function, the action of the Weyl group produces, in particular, transformation formulas for the Gauss hypergeometric function and its confluent type functions, which we called the symmetry of the hypergeometric functions [4]. Here we establish the analogous fact for the general Schlesinger systems. It will explain, for example, why the number of parameters contained in the Painlevé equations decreases after degeneration.

\subsection{Weyl group $W_{\lambda}$}

Definition 6.1. For a partition $\lambda$ of $N+1, W_{\lambda}=N_{G}\left(H_{\lambda}\right) / H_{\lambda}$ is called the Weyl group associated with $H_{\lambda}$.

Note that, when $\lambda=(1, \ldots, 1)$, the Weyl group $W_{\lambda}$ for $H_{\lambda}$ is nothing but the usual Weyl group of $G$. For the structure of $W_{\lambda}$ with general partition $\lambda$ of $N+1$, we recall necessary results from [4]. We use a different notation for partitions of $N+1$ from that used in the preceding sections. Let $\lambda$ 
be written as

$$
\lambda=(\overbrace{n_{1}, \ldots, n_{1}}^{p_{1}}, \ldots, \overbrace{n_{s}, \ldots, n_{s}}^{p_{s}})
$$

with $n_{k} \neq n_{l}(k \neq l)$. Then

$$
H_{\lambda}=\prod_{k=1}^{s} J\left(n_{k}\right)^{p_{k}}
$$

We see that the Weyl group is a semi-direct product of its continuous subgroup and its finite subgroup. To describe the continuous part of $W_{\lambda}$, we first define the continuous subgroup $W(n)$ of $\mathrm{GL}_{n}(\mathbb{C})$ as follows. Put

$$
f(T)=c_{1} T+c_{2} T^{2}+\cdots+c_{n-1} T^{n-1}, c_{1} \neq 0
$$

and

$$
f(T)^{i} \equiv w_{i, 0} T+w_{i, 1} T^{2}+\cdots+w_{i, n-1} T^{n-1} \bmod T^{n} .
$$

Then $w_{i, j}=w_{i, j}(c)$ are determined as polynomials of $c=\left(c_{1}, \ldots, c_{n-1}\right)$. Notice that $w_{i, j}=0$ for $i \geq j$ and $w_{i, i}=c_{1}^{i} \neq 0$. Set

$$
w(c)=\left(w_{i, j}(c)\right)=\left(\begin{array}{ccccc}
1 & 0 & 0 & \ldots & 0 \\
& w_{1,1} & w_{1,2} & \ldots & w_{1, n-1} \\
& w_{2,2} & \ldots & w_{2, n-1} \\
& & & \ddots & \vdots \\
& & & & w_{n-1, n-1}
\end{array}\right) \in \mathrm{GL}_{n}(\mathbb{C}) .
$$

Lemma 6.1. ([4]) The set

$$
W(n)=\left\{w(c) \mid c \in \mathbb{C}^{n-1}, c_{1} \neq 0\right\}
$$

is a subgroup of $\mathrm{GL}_{n}(\mathbb{C})$ which is isomorphic to the group of ring automorphisms $\operatorname{Aut}\left(\mathbb{C}[T] /\left(T^{n}\right)\right)$.

In order to describe the finite group part of $W_{\lambda}$, we introduce the following notation. Let $\mathscr{P}_{k}$ be the subgroup of $\mathrm{GL}_{p_{k} n_{k}}(\mathbb{C})$ consisting of permutation matrices $X$ which, when it is written blockwise as $X=\left(X_{i j}\right), X_{i j} \in \operatorname{Mat}_{n_{k}}(\mathbb{C})$, each block column and each block row contains only one nonzero block and the nonzero block is the identity matrix. Then the group $\mathscr{P}_{k}$ is isomorphic to the symmetric group $\mathfrak{S}_{p_{k}}$ by the correspondence

$$
\mathfrak{S}_{p_{k}} \ni \sigma \mapsto\left(\delta_{i \sigma(j)} I_{n_{k}}\right) \in \mathscr{P}_{k} .
$$

Example 6.1. In the case $n_{k}=2, p_{k}=3$, the group $\mathscr{P}_{k}$ consists of the following permutation matrices.

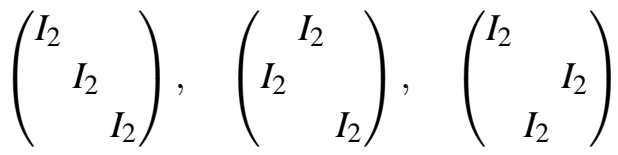

$$
\begin{aligned}
& \left(\begin{array}{cc}
I_{2} & I_{2} \\
I_{2} &
\end{array}\right), \quad\left(\begin{array}{ll}
I_{2} & \\
& I_{2} \\
I_{2} &
\end{array}\right), \quad\left(\begin{array}{ll} 
& I_{2} \\
I_{2} & \\
& I_{2}
\end{array}\right),
\end{aligned}
$$

where $I_{2}$ is the $2 \times 2$ identity matrix. 
The direct product $W\left(n_{k}\right)^{p_{k}}$ is considered as the subgroup of $\mathrm{GL}_{p_{k} n_{k}}(\mathbb{C})$ consisting of elements of the form $\operatorname{diag}\left(X_{1}, \ldots, X_{p_{k}}\right), X_{i} \in W\left(n_{k}\right)$. Then an element of $\mathscr{P}_{k}$ acts on $W\left(n_{k}\right)^{p_{k}}$ as a block permutation of diagonal blocks.

Proposition 6.1. ([4])

$$
N_{G}\left(H_{\lambda}\right)=H_{\lambda} \rtimes\left(\prod_{k=1}^{s} W\left(n_{k}\right)^{p_{k}} \rtimes \mathscr{P}_{k}\right)
$$

where $\prod_{k=1}^{s} W\left(n_{k}\right)^{p_{k}} \rtimes \mathscr{P}_{k}$ is the subgroup of $\mathrm{GL}_{N+1}(\mathbb{C})$ consisting of block diagonal matrices of $s$ blocks whose $k$-th block diagonal component belongs to $W\left(n_{k}\right)^{p_{k}} \rtimes \mathscr{P}_{k}$.

\section{Corollary 6.1.}

$$
W_{\lambda} \simeq \prod_{k=1}^{s} W\left(n_{k}\right)^{p_{k}} \rtimes \mathscr{P}_{k} .
$$

\subsection{Action of $W_{\lambda}$ on the general Schlesinger systems}

Let $W_{\lambda}$ act on $Z_{\lambda}$ by the right multiplication of matrices: $Z_{\lambda} \times W_{\lambda} \ni(z, w) \mapsto z w \in Z_{\lambda}$. It is easy to see that the action is well defined. According to the expression (6.1) of $\lambda$, we express $z \in Z_{\lambda}$ as $z=\left(z^{(1)}, \ldots, z^{(s)}\right)$ with

$$
z^{(k)}=\left(z^{(k, 1)}, \ldots, z^{\left(k, p_{k}\right)}\right), z^{(k, m)}=\left(z_{0}^{(k, m)}, \ldots, z_{n_{k}-1}^{(k, m)}\right) \in \operatorname{Mat}_{2, n_{k}}(\mathbb{C}) .
$$

Then the connection in Theorem 3.2 is locally expressed as

$$
\nabla_{\lambda}(A)=d-\sum_{k=1}^{s} \omega^{(k)}, \quad \omega^{(k)}=\sum_{m=1}^{p_{k}} \sum_{\alpha=0}^{n_{k}-1} A_{\alpha}^{(k, m)}(z) d \theta_{\alpha}\left(\vec{\zeta} z^{(k, m)}\right)
$$

which is determined by the connection matrices $A_{\alpha}^{(k, m)} \in \mathfrak{g l}_{r}(\mathbb{C})$ (here we changed the notation for the connection by omitting tilde). We array the matrices $A_{\alpha}^{(k, m)}$ according to the expression (6.3) as $A=\left(A^{(1)}, \ldots, A^{(s)}\right)$ with

$$
A^{(k)}=\left(A^{(k, 1)}, \ldots, A^{\left(k, p_{k}\right)}\right), \quad A^{(k, m)}=\left(A_{0}^{(k, m)}, \ldots, A_{n_{k}-1}^{(k, m)}\right) .
$$

Here $A$ is considered as a row vector with $N+1$ entries $A_{\alpha}^{(k, m)}$. We study the effect of the action of $W_{\lambda}$ on the set $\mathscr{A}$ of connection matrices $A$.

Definition 6.2. The action of $w \in W_{\lambda}$ on $\mathscr{A}$ is defined by

$$
A(z) \mapsto w(A)=A\left(z w^{-1}\right) \cdot{ }^{t} w^{-1} .
$$

Here the multiplication of $A$ and ${ }^{t} w^{-1}$ is considered as that of an $N+1$ dimensional row vector and a matrix of order $N+1$. 
Example 6.2. When $\lambda=(1,1,1,1), W_{\lambda} \simeq \mathfrak{S}_{4}$ consists of permutation matrices of size 4 and the connection matrices $A$ is a row vector with four entries belonging to $\mathfrak{g l}_{r}(\mathbb{C})$ :

$$
A=\left(A^{(1)}, \ldots, A^{(4)}\right), \quad A^{(k)} \in \mathfrak{g l}_{r}(\mathbb{C}) .
$$

Let $w$ correspond to $\sigma \in \mathfrak{S}_{4}$, then noting that $w={ }^{t} w^{-1}$,

$$
w(A)=\left(A^{(1)}, \ldots, A^{(4)}\right) \cdot w=\left(A^{(\sigma(1))}, \ldots, A^{(\sigma(4))}\right) .
$$

Theorem 6.1. By the change of variable $z \mapsto \tilde{z}=z \cdot w$, defined by $w \in W_{\lambda}$, the connection $\nabla_{\lambda}(A)$ for $(z, A)$ is transformed to the connection $\nabla_{\lambda}(w(A))$ for $(\tilde{z}, w(A))$.

Proof. At first, we prove the theorem for $w \in \mathscr{P}_{k}$. Assume that $w$ corresponds to a permutation $\sigma \in \mathfrak{S}_{p_{k}}$. Then $\tilde{z}=z \cdot w$ reads

$$
\tilde{z}^{(l)}= \begin{cases}z^{(l)}, & l \neq k, \\ \left(z^{(k, \sigma(1))}, \ldots, z^{\left(k, \sigma\left(p_{k}\right)\right)}\right), & l=k .\end{cases}
$$

Hence $\nabla_{\lambda}(A)=d-\sum_{l} \omega^{(l)}$ with

$$
\omega^{(l)}=\sum_{m=1}^{p_{l}} \sum_{\alpha=0}^{n_{l}-1} A_{\alpha}^{(l, m)}(z) d \theta_{\alpha}\left(\vec{\zeta}_{z}\right)
$$

is written, for $l \neq k$, as

$$
\omega^{(l)}=\sum_{m} \sum_{\alpha} A_{\alpha}^{(l, m)}\left(\tilde{z} w^{-1}\right) d \theta_{\alpha}\left(\vec{\zeta} \tilde{z}^{(l, m)}\right)
$$

and, for $l=k$, as

$$
\begin{aligned}
\omega^{(k)} & =\sum_{m} \sum_{\alpha} A_{\alpha}^{(k, m)}\left(\tilde{z} w^{-1}\right) d \theta_{\alpha}\left(\vec{\zeta} \tilde{z}^{\left(l, \sigma^{-1}(m)\right)}\right) \\
& =\sum_{m} \sum_{\alpha} A_{\alpha}^{(k, \sigma(m))}\left(\tilde{z} w^{-1}\right) d \theta_{\alpha}\left(\vec{\zeta} z^{(l, m)}\right) .
\end{aligned}
$$

Thus $\nabla_{\lambda}(A)$ is taken to $\nabla_{\lambda}(w(A))$ by the change of variables $z \mapsto \tilde{z}=z w$.

Next we prove the theorem in the case where $w$ is an element of the continuous part $\prod_{k} W\left(n_{k}\right)^{p_{k}}$. Since its each $(k, m)$-th factor $W\left(n_{k}\right)$ acts only on $(k, m)$-th factor $z^{(k, m)}$ of $z$, we can concentrate our consideration on each $(k, m)$-th factor. Hence we can omit the suffix $(k, m)$ indexing the block structure and we are led to the situation where $\lambda=(n), H_{\lambda}=J(n)$ and $W_{\lambda}=W(n)$. Then the connection form $\omega$ is locally written as

$$
\begin{aligned}
\omega & =\sum_{\alpha=0}^{n-1} A_{\alpha}(z) d \theta_{\alpha}(\vec{\zeta} z) \\
& =\sum_{\alpha} A_{\alpha}\left(\tilde{z} w^{-1}\right) d \theta_{\alpha}\left(\vec{\zeta} \tilde{z} w^{-1}\right) \\
& =\left(d \theta_{0}\left(\vec{\zeta} \tilde{z} w^{-1}\right), \ldots, d \theta_{n-1}\left(\vec{\zeta} \tilde{z} w^{-1}\right)\right)\left(\begin{array}{c}
A_{0}\left(\tilde{z} w^{-1}\right) \\
\vdots \\
A_{n-1}\left(\tilde{z} w^{-1}\right)
\end{array}\right),
\end{aligned}
$$

where the multiplication of the last line is understood as a matrix multiplication in a similar sense as in Def. 6.2. 
Lemma 6.2. ([4], p31) For $w \in W(n)$ and $h \in J(n)$, we have

$$
\left(\theta_{0}(h w), \ldots, \theta_{n-1}(h w)\right)=\left(\theta_{0}(h), \ldots, \theta_{n-1}(h)\right) \cdot w .
$$

By virtue of the above lemma, we see that $\omega$ in (6.5) is taken to

$$
\omega=\left(d \theta_{0}(\vec{\zeta} \tilde{z}), \ldots, d \theta_{n-1}(\vec{\zeta} \tilde{z})\right) w^{-1}\left(\begin{array}{c}
A_{0}\left(\tilde{z} w^{-1}\right) \\
\vdots \\
A_{n-1}\left(\tilde{z} w^{-1}\right)
\end{array}\right)
$$

This implies that the connection matrix $A=\left(A_{0}, \ldots, A_{n-1}\right)$ is taken to

$$
w(A)=\left(A_{0}\left(\tilde{z} w^{-1}\right), \ldots, A_{n-1}\left(\tilde{z} w^{-1}\right)\right)^{t} w^{-1} .
$$

This completes the proof of Th. 6.1.

Corollary 6.2. If the connection $\nabla_{\lambda}(A)$ is flat, so is $\nabla_{\lambda}(w(A))$ for any $w \in W_{\lambda}$.

Proof. The assertion is evident since $\nabla_{\lambda}(w(A))$ is obtained from $\nabla_{\lambda}(A)$ by the change of independent variables $z \rightarrow \tilde{z}=z \cdot w$.

\section{Remark 6.1.}

1. Cor. 6.2 implies that $\nabla_{\lambda}(w(A))$ describes an isomonodromic deformation if does $\nabla_{\lambda}(A)$.

2. Let $X_{\lambda}$ be a realization of the quotient space $\mathrm{GL}_{2}(\mathbb{C}) \backslash Z_{\lambda} / H_{\lambda}$ as in the examples of Painlevé equations. Then the action of $W_{\lambda}$ on $Z_{\lambda}$ induces that on $X_{\lambda}$. Using Th. 6.1, this action on $X_{\lambda}$ gives a symmetry of Painlevé equations, for example.

\subsection{Effect of the continuous part of $W_{\lambda}$}

The effect of the action of finite part of $W_{\lambda}$ on the connection matrices $A$ of $\nabla_{\lambda}(A)$ is already illustrated in Example 6.2 and evident. Namely, for $w \in \mathscr{P}_{k}$ corresponding to $\sigma \in \mathfrak{S}_{p_{k}}$, then

$$
w(A)^{(l, m)}= \begin{cases}A^{(l, m)}, & \text { if } l \neq k \\ A^{(k, \sigma(m))}, & \text { if } l=k .\end{cases}
$$

Next we consider the effect of the continuous part $W_{c}=\prod_{k=1}^{s} W\left(n_{k}\right)^{p_{k}}$ on the general Schlesinger systems.

Proposition 6.2. By the action of $W_{c} \subset W_{\lambda}$, we can reduce $N+1-\ell(\lambda)$ parameters in the connection form of $\nabla_{\lambda}(A)$, where $\ell(\lambda)$ is the number of parts of $\lambda$.

Proof. In the action $A \mapsto w(A)$ of $w \in W_{c}$, the $(k, m)$-th factor $W\left(n_{k}\right)$ acts only on the $(k, m)$-th factor $A^{(k, m)}=\left(A_{0}^{(k, m)}, \ldots, A_{n_{k}-1}^{(k, m)}\right)$ of $A$, so we can restrict our discussion to the situation where 
$\lambda=(n), W_{\lambda}=W(n)$ and the connection form is locally written as

$$
\omega=\sum_{0 \leq \alpha<n} A_{\alpha}(z) d \theta_{\alpha}(\vec{\zeta} z)
$$

and hence $A=\left(A_{0}, \ldots, A_{n-1}\right)$. Let $A \in \mathscr{A}$ is given and consider the action of $v \in W(n)$. Put

$$
v^{-1}=w(c)=\left(w_{i, j}(c)\right)=\left(\begin{array}{ccccc}
1 & 0 & 0 & \ldots & 0 \\
& w_{1,1} & w_{1,2} & \ldots & w_{1, n-1} \\
& w_{2,2} & \ldots & w_{2, n-1} \\
& & \ddots & \vdots \\
& & & w_{n-1, n-1}
\end{array}\right) .
$$

Then the transformed connection matrix $B=w(A)$ is given by

$$
B:=A \cdot{ }^{t} v^{-1}=\left(A_{0}, \ldots, A_{n-1}\right)\left(\begin{array}{c|ccc}
1 & 0 & \ldots & 0 \\
\hline 0 & w_{1,1} & & \\
\vdots & \vdots & \ddots & \\
0 & w_{1, n-1} & \ldots & w_{n-1, n-1}
\end{array}\right) .
$$

It follows that

$$
\begin{aligned}
B_{n-1} & =A_{n-1} w_{n-1, n-1}, \\
B_{n-2} & =A_{n-2} w_{n-2, n-2}+A_{n-1} w_{n-2, n-1}, \\
& \vdots \\
B_{1} & =A_{1} w_{1,1}+A_{2} w_{1,2}+\cdots+A_{n-1} w_{1, n-1}, \\
B_{0} & =A_{0} .
\end{aligned}
$$

On the other hand, we see from (6.2) that

$$
w_{k, n-1}= \begin{cases}k c_{1}^{k-1} c_{n-k}+\left(\text { a polynomial of } c_{1}, \ldots, c_{n-1-k}\right), & 1 \leq k<n-1 \\ c_{1}^{n-1}, & k=n-1\end{cases}
$$

and $w_{k, m}(k \leq m<n-1)$ are polynomials of $c_{1}, \ldots, c_{n-1-k}$. It follows that we can decreases the number of parameters of $A$ by $n-1$ by determining $c$ appropriately.

We shall illustrate the effect of action of $W_{c}$ by considering, as an example, the Painlevé $V$ case, which is treated in Sec. 5.

In this case the Weyl group is $W \simeq W(2) \times \mathfrak{S}_{2}$ whose elements are

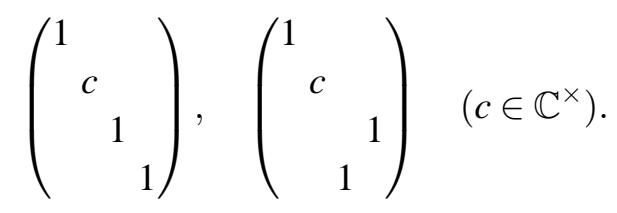

Hence $W_{c}=W(2)$ and $\operatorname{dim} W_{c}=1$. We assert that we can decrease the number of constants in GSS corresponding to $P_{V}$. As is seen in Section 5, GSS is obtained as an integrability condition of the 
connection

$$
\Phi^{*} \nabla=d-\left(A_{1}(t) d \zeta+A_{2}(t) d \log \zeta+A_{3}(t) d \log (\zeta+t)\right),
$$

which gives an isomonodromic deformation of the linear equation on $\mathbb{P}^{1}$

$$
\frac{d y}{d \zeta}=\left(A_{1}+\frac{A_{2}}{\zeta}+\frac{A_{3}}{\zeta+t}\right) y
$$

It has 2 regular singular points $\zeta=0,-t$ and an irregular singular point $\zeta=\infty$. Let us look at $\zeta=\infty$ in (6.10) using a local coordinate $s=1 / \zeta$. Eq. (6.10) can be written as

$$
\frac{d y}{d s}=\left(-\frac{A_{1}}{s^{2}}+\frac{A_{0}}{s}+(\text { holomorphic at } s=0)\right) y,
$$

where $A_{0}=-A_{2}-A_{3}$. If we assume $A_{1}$ has two distinct eigenvalues $\alpha_{1}, \alpha_{2}$, then (6.11) can be reduced, by local gauge transformation $y \mapsto z$, to

$$
\frac{d z}{d s}=\left(-\frac{\tilde{A}_{1}}{s^{2}}+\frac{\tilde{A}_{0}}{s}+(\text { holomorphic at } s=0)\right) z
$$

with diagonal matrices

$$
\tilde{A}_{1}=\left(\begin{array}{cc}
\alpha_{1} & \\
& \alpha_{2}
\end{array}\right), \quad \tilde{A}_{0}=\left(\begin{array}{cc}
\alpha_{1}^{\prime} & \\
& \alpha_{2}^{\prime}
\end{array}\right)
$$

Then we know that $\tilde{A}_{1}, \tilde{A}_{0}$ are constants of integration of GSS obtained from $\Phi^{*} \nabla$. As is seen in (6.7) in the proof of Prop. 6.2, the effect of $\operatorname{diag}(1, c) \in W(2)$ is

$$
A_{1} \mapsto B_{1}=A_{1} c .
$$

This induces the change of constants of integration $\left(\alpha_{1}, \alpha_{2}\right) \mapsto\left(\alpha_{1} c, \alpha_{2} c\right)$. Since one of $\alpha_{1}, \alpha_{2}$ is not zero at least, say $\alpha_{1}$, then we can normalize $\alpha_{1}$ to any desired nonzero value, say 1 . Thus the number of constants of integration of GSS is decreased by 1 .

\section{Acknowledgments}

We would like to thank the referee for valuable comments. This work is supported in part by JSPS grants-in-aid No.23540247.

\section{References}

[1] B. Gambier, Sur les équations différentielles du second ordre et du premier degré dont l'integrale générale est à points critiques fixes, Acta. Math. Ann. 33, (1910), 1-55.

[2] N. Hitchin, Geometrical aspects of Schlesinger's equation. J. Geom. Phys. 23 (1997), no. 3-4, 287-300.

[3] K. Iwasaki, H. Kimura, S. Shimomura and M. Yoshida, From Gauss to Painlevé, Vieweg Verlag, (1991).

[4] H. Kimura, T. Koitabashi, Normalizer of maximal abelian subgroup of $G L(n)$ and general hypergeometric functions. Kumamoto J. Math. 9 (1996), 13-43.

[5] L.J.Mason, N.M.J. Woodhouse, Self-duality and the Painlevé transcendents, Nonlinearity 6 (1993), $569-581$.

[6] L.J.Mason, N.M.J. Woodhouse, Twistor theory and the Schlesinger equations, Applications of analytic and geometric methods to nonlinear differential equations (Exeter, 1992), 17-25, NATO Adv. Sci. Inst. Ser. C Math. Phys. Sci., 413, Kluwer Acad. Publ., Doldrecht, (1993). 
[7] L.J.Mason, N.M.J. Woodhouse, Integrability, Self-Duality and Twistor Theory, Oxford Univ. Press, (1996)

[8] Y. Murata, Private communication.

[9] M. Noumi, Painlevé equations through symmetry, Translations of Mathematical Monographs, 223, Amer. Math. Soc., Providence (2004).

[10] Y. Ohyama, Isomonodromy deformations and twistor theory, Contemporary Math. 309, (2002), 185193.

[11] P. Painlevé, Mémoire sur les équations différentielles dont l'intégrale générale est uniforme, Bull. Soc. Math. France 28, (1900), 201-261.

[12] L. Schlesinger, 灼 ber eine Klasse von Differentialsystemen beliebiger Ordnung mit festen kritischen Punkten, J. Reine Angew. Math. 141,(1912),96-145.

[13] C. Tracy, H. Widom, Random unitary matrices, permutations and Painlevé, Comm. Math. Phys. 207, (1999), no. 3, 665-685. 